\title{
Development of an Enhanced Fuzzy C-Means algorithm for Medical Image Segmentation using Ant Colony Optimization
}

\author{
${ }^{1}$ Ajala F.A, ${ }^{2}$ Ojebamigbe V.I, , ${ }^{3}$ Fenwa O.D \\ ${ }^{123}$ Department of Computer Science and Engineering, LadokeAkintola University of Technology, P.M.B. \\ 4000, Ogbomoso. \\ Corresponding Author E mail: faajala@lautech.edu.ng, odfenwa@lautech.edu.ng,
}

\begin{abstract}
Modified Fuzzy C-Means (MFCM) algorithm, which is a combination of fuzzy c means and k means, for medical image segmentation, suffers from high computational time and noise which lead to high memory consumption and low accuracy. Therefore, this paper presents an Enhanced MFCM algorithm (ACOMFCM), which has better accuracy and low memory consumption for medical image segmentation. Thirty medical images used in this work were pre-processed using Gaussian filtering method. ACOMFCM algorithm was developed using Ant Colony Optimization techniques to minimise the Euclidean distance between the data point and centre coordinate in the K-means algorithm characterizing the existing MFCM. Segmentation of the thirty images were carried out using the MFCM and ACOMFCM algorithms in Matrix Laboratory 7.1 (R0011a) environment. Performance of the MFCM and the ACOMFCM was evaluated using segmentation accuracy, segmentation time and memory consumption.

The average result of MFCM algorithm for thirty images yielded segmentation accuracy, segmentation time and memory consumption of $7117992.29,22.484 \mathrm{~s}$ and 494161920bit, respectively while average result of the ACOMFCM algorithm for thirty images used yielded segmentation accuracy, segmentation time and memory consumption of $10590135.79,6.649 \mathrm{~s}$ and 502960128 bit, respectively.
\end{abstract}

Keywords: MFCM, ACOMFCM, Segmentation, Accuracy, Memory Consumption.

\section{Introduction}

Image segmentation is a means of dividing digital image into different regions to get useful information. The role of Segmentation cannot be underestimated in image analysis as it is one of the peculiar challenges in image processing technology because Clinicians and Patients always 
desire quality images. The images are divided into non-overlapped and consistent regions which are similar with regard to some image properties such as intensity, color, texture and so on (Krishnan and Ramamoorthy, 2014).

$\mathrm{Yu}$, Lee, Jeon (2014) reported that image segmentation has a wide range of applications such as image content analysis, object recognition and computer-assisted medical diagnosis. Researcherson Image segmentation have delivered a considerable progress over the year, yet there have not been a perfect algorithm for segmentation. According to Dasgupta, 2012, the arrival of Computed Tomography (CT), Positron Emission Tomography (PET), Magnetic Resonance Imaging (MRI) and other peculiar medical image modalities has significantly improved the diagnosis of diseases in human beings. Medical imaging gives a dependable source of information of the human body, both to the Patient and clinician for use in fields like: reparative surgery, radiotherapy, stereotactic etc.

Hamed and Hadi, (2012) reported that minimizing the weighted vector within group sum of imagesis achievable through Fuzzy $\mathrm{C}$ means (FCM) algorithm as it produces a C-partitioning method in an iterative clustering format. FCM allows a pixel to be in multiple clusters with different degree of membership function. According to Krishnan and Ramamoorthy, (2014); Ghizlane, Amina and Khalid (2013), Ant Colony Optimization (ACO) is a meta-heuristic scheme which was used for solving discrete swarm optimization problemsand handle the optimization and clustering problems. Due to its parallel searching capability, artificial ants implement a randomized construction heuristic which makes probabilistic decisions.

\section{Literature Review}

Segmentation is divided into different types with their unique peculiarities: According to Rubal Ahuja and Krishan Kumar, (2014); Nitesh et al, (2014); Joyjit et al, (2014); Sasibala and Sudhavani, (2016), Segmentation can be divided into Edge based, Model based, Thresholding, Region based and Clustering. Fuzzy c meams, one the methods under clustering has been modified by Ajalaetal (2012) to overcome its inability to segment colour images.

\subsection{Modified Fuzzy C-Means (MFCM) Algorithm}

MFCM is an approach developed by Ajalaetal (2012) where the data points are updated iteratively. It worked on colored images by the incorporation of K-means algorithm. The strength of K-means (ability to work on colored images) and FCM (allowing the member of one cluster to belong to another member) was combined to get MFCM algorithm. The step for the MFCM algorithm is shown as thus;

Step 1: Input the image to be segmented 
Step 2: Get the number of colors in the image by looking for the $a^{*} b^{*}$ space

Step 3: Assign the number of colors to be cluster cluster no; that is $C=$ no of colors

Step 4: Choose an appropriate level of cluster fuzziness $f>1$.

Step 5: Initialize the $N \times C \times M$ sized membership matrix $U$, at random,

Step 6: Determine the cluster centersCCjm, for jth cluster and its mth dimension by using the expression given below:

$$
\frac{\sum_{i=1}^{N} U_{i j m}^{f} x_{i m}}{\sum_{i=1}^{N} U_{i j m}^{f}}
$$

Step 7: Calculate the Euclidean distance between ith data point and jth cluster center using repetitive structure

Step8: Update fuzzy membership grade point $n$ according.

Step 9: Repeat from Step 6 to Step 8 until the changes a pre-specified termination criterion is reached

Stop 10: Stop and output the image.

\subsection{Ant Colony Optimization (ACO)}

Ant colony algorithm used byDohyeun Kim, (2016) and Cheraghali, et al (2014) was used in conjunction with modified MFCM algorithm that was developed by Ajala et al (2012) to form ACOMFCM. The ACO algorithm is presented as follows;

Step 1: Initialize all parameters, $n$ fault symptom samples can be regarded as $n$ classes.

Step 2: Compute weighted Euclidean distance between samples Xi and Xj.

$$
d i j=\left\|D\left(X_{i}-X_{j}\right)\right\|^{2}=\sqrt{\sum_{k=1}^{m} P_{k}\left(X_{i k}-X_{j k}\right)^{2}} \quad 3.6
$$

Pkis weighted factor which can be defined according to every component's contribution in clustering.

Step 3: Compute the pheromone quantity of trail, $r$ is described as cluster center and $\tau_{i j(t)}$ is intensity of the pheromone trail between $\mathrm{Xi}$ and $\mathrm{Xj}$ at time $t$, set the intensity of pheromone trail to 0 at time 0 .

$O d i j>r$

$$
\tau_{i j}(t)=\{1 d i j \leq r
$$

Step 4: Compute the probability of merging class Xi and Xj.

$$
P_{i j}=\frac{\tau_{i j}(t)^{\alpha} \cdot \mu_{i j}(t)^{\beta}}{\sum \tau_{i s}(t)^{\alpha} \cdot \mu_{i s} \beta}
$$

Where $S\{s \mid d r, s 1,2, \ldots . ., n\}$ is $=_{-}=$and $\eta i j(t)$ is weight coefficient. It can reflect expectation of mergering class $X i$ and $X j$.

Step 5: If Pij $(t) \geq P 0$ merging $X i$ and $X j$, computing cluster center after merging. 
$\overline{C_{J}}=\frac{1}{J} \sum_{k=1}^{j} X_{k}\left(X_{K} \in C_{J}\right)$

Step 6: Compute the biased error of the jthclustering.

$$
D_{j}=\sum_{k=1}^{j} \sqrt{\sum_{i=1}^{m} X_{k i}-C_{j i}^{2}}
$$

Where cji is the ith component of the jth cluster center.

Step 7: Compute overall error

$$
\in=\sum_{J=1}^{K} D_{j}, \text { if } \in \leq \epsilon_{0}
$$

The whole process will pause. Please output the number of clustering $m$ and cluster centerj c Otherwise, jump step 3 and continue iteration.

\section{Step 8: Output image}

Step 9: Stop

\subsection{Related Work}

Dasgupta (2012), performed demarcation of brain tumor using modified fuzzy c-means. The area of the segmented tumor generated from the modified fuzzy c-means segmentation was evaluated using Edge-adaptive clustering model. Despite this modification, the system still has the problem of Long Computational Time and Memory Consumption.

Ajala et al (2012) worked on Modified Fuzzy C-Means for Colored Image Segmentation (continuation of the Fuzzy k-c-means Clustering Algorithm for Medical Image Segmentation). The research describes FCM as a method of clustering which allows one piece of data to belong to two or more clusters. The results shows better performance than the existing fuzzy algorithm in terms of cluster centers, simulation time, iteration count and the outputted image. However, when applied to medical image analysis, it has important drawbacks such as long computational time and sensitivity to noise which lead to high memory consumption.

Lucia Ballerni, Leonardo Bocchi and Carina Johansson (2013) worked on Clustering algorithm for Color image segmentation. The classical Fuzzy C-Means algorithm was used with a Genetic algorithm (GA). From the result, segmentation of noise images is effectively improved. However, the limitation of the system occurs in long computational time.

\section{Methodology}

The methodology adopted were as follows

\section{(a) Image Acquisition}

Thirty medical images comprising of brain, eyes, finger, foetus, leg etc were used for the work Sample images were depicted in figure 3.1

\section{(b) Image Pre-processing}


Gaussian Filtering algorithm (GFA) was used to pre-process the acquired images and to prepare the images for further processing.

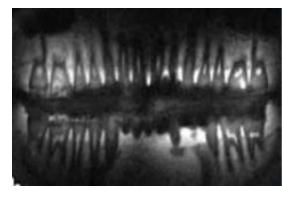

Teeth
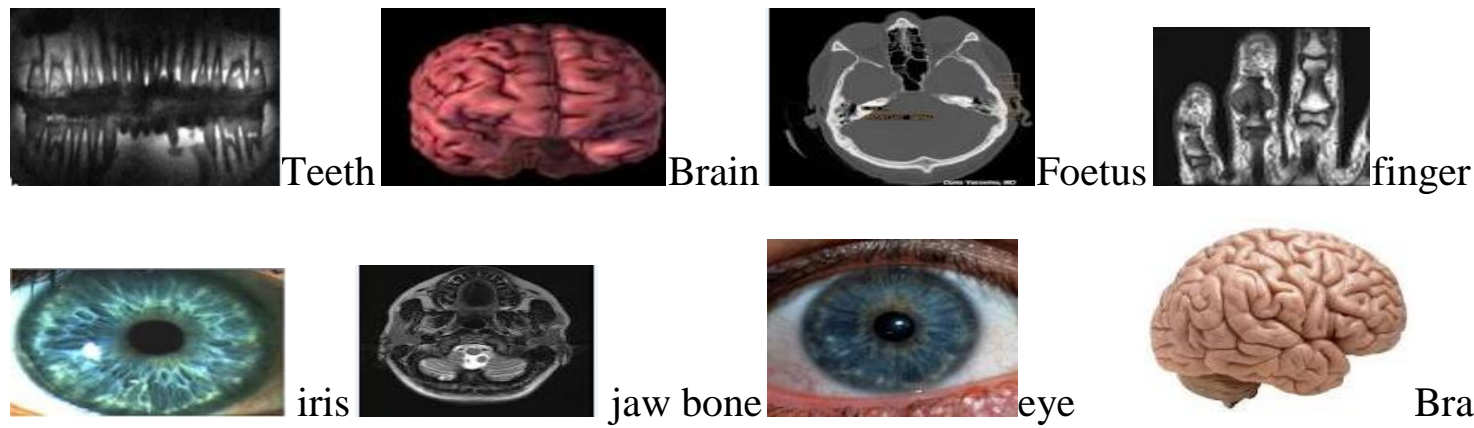

jaw bone
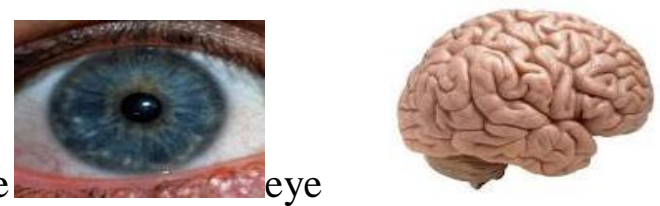

Brain

Figure 3.1: Samples of the medical images used

\section{(c) Ant Colony Optimization with Modified Fuzzy C Means (ACOMFCM)}

The Modified FCM algorithm method is optimized using Ant Colony Optimization (ACO) algorithm, known as ACOMFCM. ACO depicts an ant that move randomly from their colony in search of food and after gotten the food they create a marker or pheromone that forms the shortest distance between the Colony and the food source. This selection ability of ACO was employed in MFCM to get the shortest distance between cluster center and the data $\mathrm{n}$ point in each cluster. ACO helps to further reduce time and noise by its ability of picking through the shortest distance. The resulting algorithms (ACOMFCM) produces high quality segmented image. The step for the ACOMFCM algorithm is shown thus;

1. Input the image to be segmented

2. If image $=$ colored image goto 3 else goto 4

3. Load K means clustering segmentation and goto 4

4. Generate initial fuzzy partitioning /membership matrix

$$
n_{i j}=\frac{1}{\sum_{k=1}^{c} \frac{\left\|x_{1}-c_{j 1}\right\|^{\frac{2}{m-1}}}{\left\|x_{1} c_{k}\right\|}}
$$

5. Calculate initial fuzzy cluster

6. Calculate the cluster centroid

$$
C=\frac{\sum_{i=1}^{n} U_{i j}^{m}{ }^{m} x_{i}}{\sum_{i=1}^{n} U_{i j}^{m}}
$$

7. Locate the membership grade point (n) in the cluster

8. Start the iteration using Ant Colony Optimization (ACO) for selection process

(Hint: ACO was used to find near optimal combination between $c$ and $n$ )

9. Get the probability of ant movement from $c$ to $n$ that depends on attractiveness and trail level. 


$$
P_{c n}^{K}=\frac{\left(\boldsymbol{T}_{c n}^{\alpha}\right)\left(\eta_{c n}^{\beta}\right)}{\sum_{x y}\left(\boldsymbol{T}_{z c}^{\alpha}\right)\left(\eta_{z c}^{\beta}\right)}
$$

Where;

$T_{c n}=$ amount of pheromone deposited for transition from $c$ to $n$

$\eta_{c n}=$ desirability of cluster transition for $\mathrm{cn}\left(1 / d_{c n}, d=\right.$ distance $)$

$T_{z c}=$ attractiveness for other possible cluster transition

$\eta_{z c}=$ trail level for other possible cluster transition

$0 \leq \alpha=$ parameter to control the influence of $T_{c n}$

$\beta \geq 1=$ parameter to control influence of $\eta_{c n}$

10. If $n=0$, go to 11 , else go to 9

11. Update pheromone when ants have completed the solution using

$(1-P) T_{c n}+\sum \Delta T_{c n}^{k}$

Where

$\sum \Delta T_{c n}^{k}=Q / L_{k}$ if ant uses curve $c n$ in its tour, otherwise 0

$L_{k}=$ length of ant tour and $Q=$ constant

12. Defuzzification process then takes place in order to achieve the crisp clusters

13. Output image.

\section{(d) Performance Evaluation}

The performance evaluations of the algorithms were carried out using the metrics by Lauren (2001), which are segmentation time, segmentation accuracy and Memory consumption.

\section{Segmentation Time}

This is the time taken to compute the segmentation including the running time of the algorithm or the time spent to perform interactive segmentation. The research was carried out using Compaq Presario CQ60 notebook PC with AMD Athlon Dual-core QL 62 2.00 GHz processor. It was computed in milli seconds using computer time.

\section{Segmentation Accuracy}

The accuracy of the segmentation algorithms is generally based on Volume measurement. This is the volume of segmented feature in the image domain. It is can be evaluated using segmented volume area;

Segmented volume area $=$ Number of pixel $*$ volume of each pixel 


\section{Memory Consumption}

This depends highly on the computer memory. System performance is highly dependent on the memory being consumed. System memory is of paramount importance as it plays a major role in image processing. Obviously segmentation types with low memory consumption could be accepted, as low memory consumption would aid system performance.

\section{Result}

Table 4.1 gave the results ofsegmentation time, accuracy and memory consumption of thirty (30) medical images used to test the developed ACOMFCM segmentation algorithm.Also, Table 4.2 shows the average result of segmentation accuracy, segmentation time and memory consumption of the thirty (30) medical images used for the developed ACOMFCM segmentation algorithm.

Table 4.1: Results showing performance of thirty (30) medical images using ACOMFCM

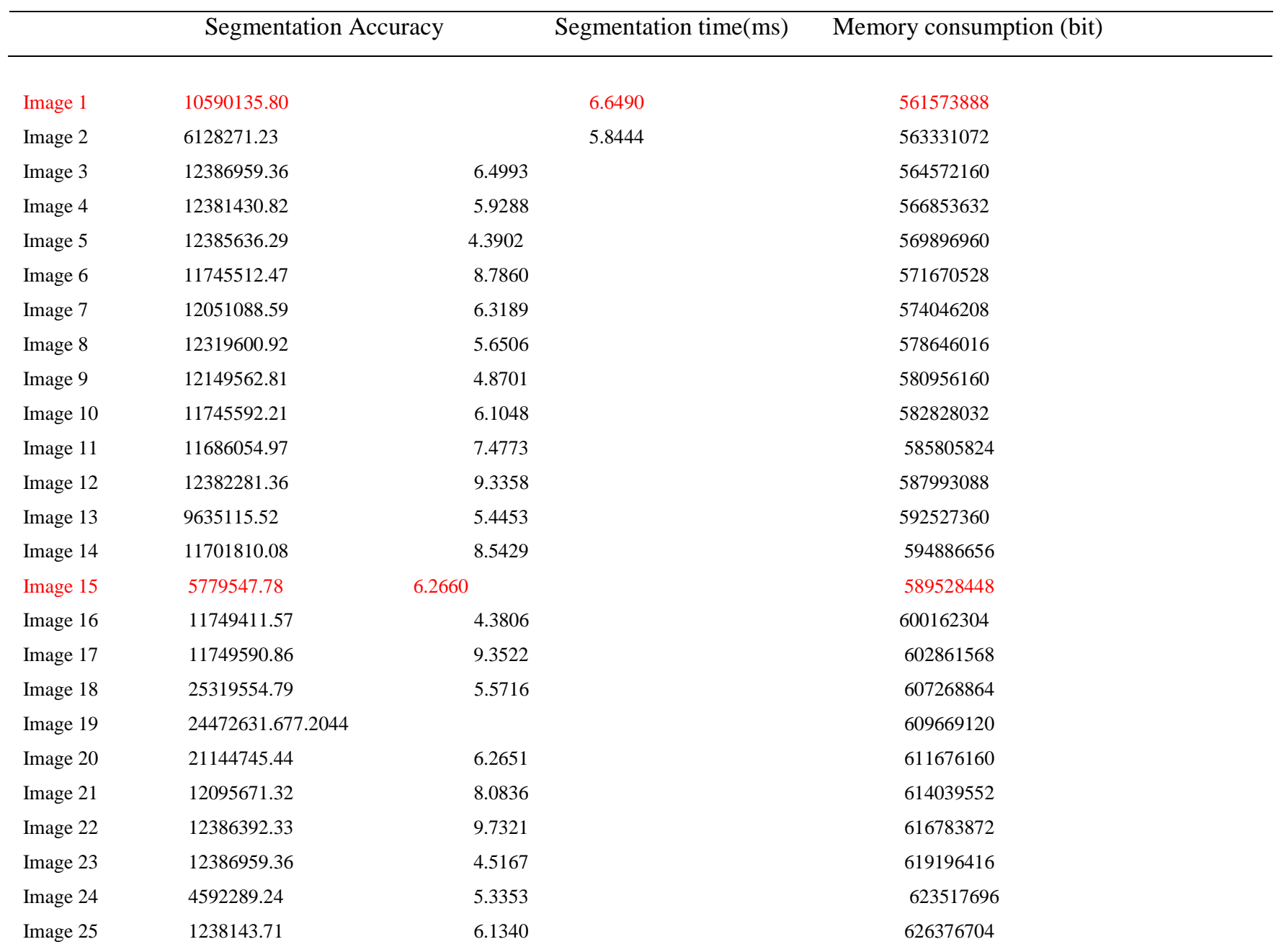




$\begin{array}{ll}9569788.93 & 8.3511 \\ 11679847.05 & 7.5672 \\ 12325365.73 & 4.7214 \\ 12363672.42 & 9.7812 \\ 12381430.755 .9288 & \end{array}$

628617216

Table 4.2: Average result of the thirty (30) medical images used for ACOMFCM

\begin{tabular}{lccc}
\hline & Segmentation Accuracy & Segmentation time(ms) & Memory consumption (bit) \\
\hline \multirow{2}{*}{ ACOMFCM } & 10590135.79 & 6.6490 & 502960128 \\
\hline
\end{tabular}

\section{Discussion of Results}

The result obtained for ACOMFCMsegmentation algorithm was compared with MFCM segmentation algorithm developed by Ajalaetal (2012). Both MFCM and ACOMFCM algorithms were tested using thirty medical images. In the analysis done, the values of segmentation accuracy, segmentation time and memory consumption were computed for both the MFCM and the ACOMFCM.Table 4.3 gave the results of segmentation time, accuracy and memory consumption of thirty (30) medical images used to test MFCM segmentation algorithmwhile Table 4.4 shows the average result of segmentation accuracy, segmentation time and memory consumption of the thirty (30) medical images used for MFCM and ACOMFCM segmentation algorithms and it can be deduced that ACOMFCM has increase segmentation accuracy of $20 \%$, decrease segmentation time of $54 \%$ and increase memory consumption of $1 \%$ compared to MFCM.

Moreover,user interface designed for MFCM and ACOMFCM segmentation algorithms for three randomly selected samples of medical images; image 1,15 and 30 were presented as shown in figure 4.1, figure 4.2 and figure 4.3.The bar chart of segmentation time, accuracy and memory consumption were presented in figure 4.4, figure 4.5 and figure 4.6. It was discovered that ACOMFCM has higher segmentation accuracy compared to MFCM and also, ACOMFCM has an increase memory consumption compared to MFCM because of the robustness of ACO algorithm. 
International Academic Conference on Applied Research in Engineering, Science \& Technology Brussels, Belgium $14^{\text {th }}-15^{\text {th }}$ September 2018

Table 4.1: Results showing performance of thirty (30) medical images using MFCM

\begin{tabular}{|c|c|c|c|}
\hline & Segmentation Accuracy & Segmentation time $(\mathrm{ms})$ & Memory consumption (bit) \\
\hline Image 1 & 71179924.29 & 22.4842 & 442568704 \\
\hline Image 2 & 5813119.67 & 17.6057 & 448299008 \\
\hline Image 3 & 11749949.44 & 14.1741 & 448704512 \\
\hline Image 4 & 11744705.21 & 30.3736 & 638954368 \\
\hline Image 5 & 11542761.38 & 20.6182 & 549265664 \\
\hline Image 6 & 11430639.64 & 12.7821 & 419433600 \\
\hline Image 7 & 11749008.17 & 15.1472 & 449613824 \\
\hline Image 8 & 11516827.62 & 25.7256 & 447242240 \\
\hline Image 9 & 11473641.14 & 18.5131 & 447188992 \\
\hline Image 10 & 10572345.23 & 32.3410 & 656892416 \\
\hline Image 11 & 11005107.25 & 19.3533 & 451350528 \\
\hline Image 12 & 11633007.54 & 35.1675 & 651452928 \\
\hline Image 13 & 9139621.51 & 15.7312 & 391274240 \\
\hline Image 14 & 9672336.92 & 31.8036 & 591233280 \\
\hline Image 15 & 54823296.34 & 34.7937 & 516284416 \\
\hline Image 16 & 10065066.02 & 27.4042 & 531222528 \\
\hline Image 17 & 11005107.25 & 20.7165 & 536039424 \\
\hline Image 18 & 12366924.31 & 29.5232 & 530395136 \\
\hline Image 19 & 12044756.76 & 16.8063 & 431664896 \\
\hline Image 20 & 12141199.11 & 30.1430 & 636940544 \\
\hline Image 21 & 11748425.48 & 21.2945 & 532828160 \\
\hline Image 22 & 10028653.38 & 27.4534 & 533164032 \\
\hline Image 23 & 6128271.23 & 15.3538 & 432844544 \\
\hline Image 24 & 1238535.78 & 20.4773 & 533401600 \\
\hline Image 25 & 1214191.11 & 17.1084 & 438329088 \\
\hline Image 26 & 4592289.24 & 28.8710 & 531267584 \\
\hline Image 27 & 11430639.45 & 14.5065 & 531230720 \\
\hline Image 28 & 10439160.25 & 19.7214 & 27424 \\
\hline Image 29 & 10842809.27 & 25.7680 & 534339584 \\
\hline Image 30 & 11744706.28 & 30.3736 & 4793964 \\
\hline
\end{tabular}


Table 4.4: Average result of the thirty (30) medical images used for MFCM and ACOMFCM

Segmentation Accuracy $\quad$ Segmentation time(ms) Memory consumption (bit)

$\begin{array}{llll}\text { MFCM } & 7117992.29 & 22.484 & 494161920\end{array}$

$\begin{array}{llll}\text { ACOMFCM } & 10590135.79 & 6.6490 & 502960128\end{array}$

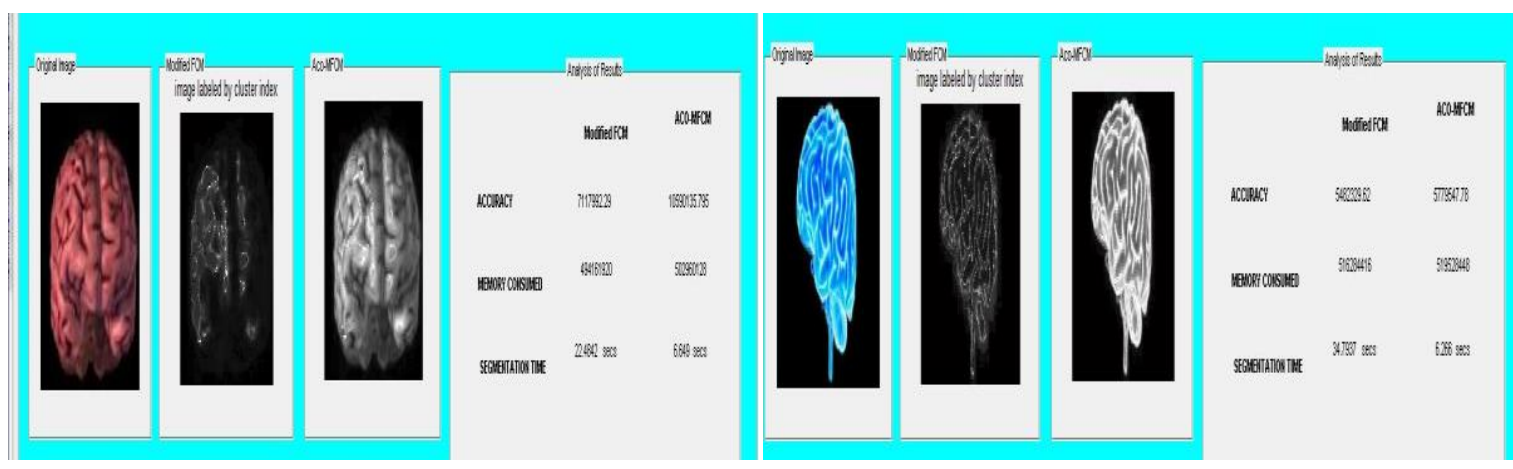

Figure 4.1: Image 1 interface for MFCM Figure 4.2: Image15 interface for MFCM and ACOMFCM and ACOMFCM

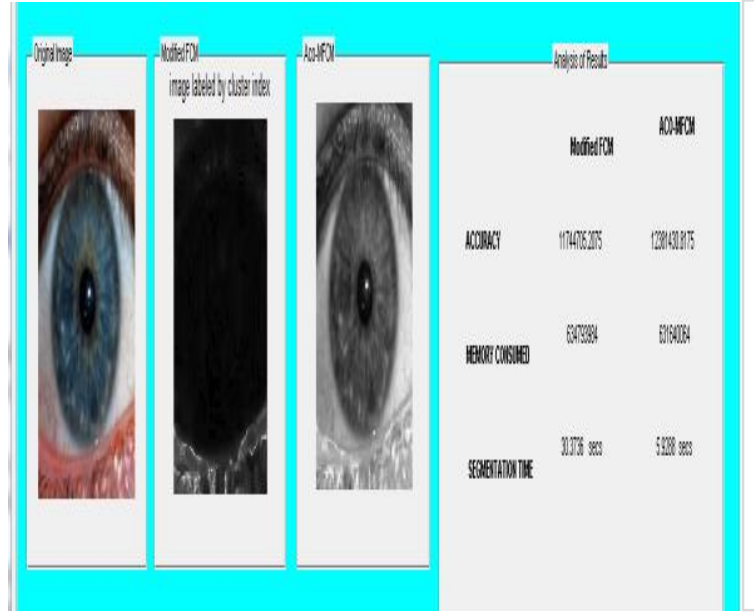

Figure 4.3: Image 30 interface for MFCM and ACOMFCM

\section{Segmentation time for MFCM} and ACOMFCM

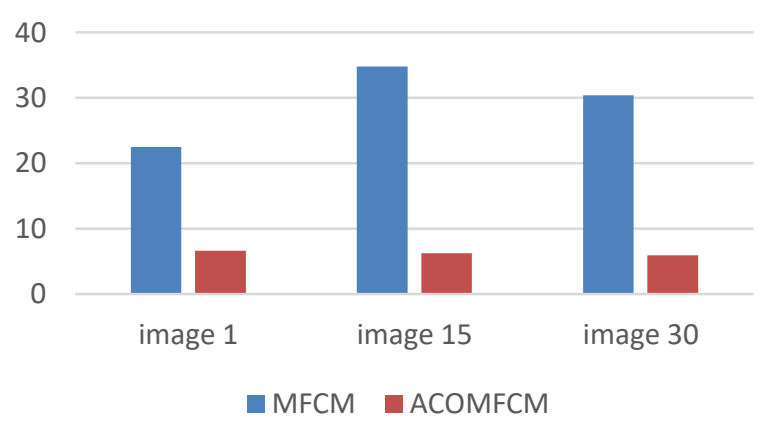

Figure 4.4: Segmentation time for MFCM and ACOMFCM 


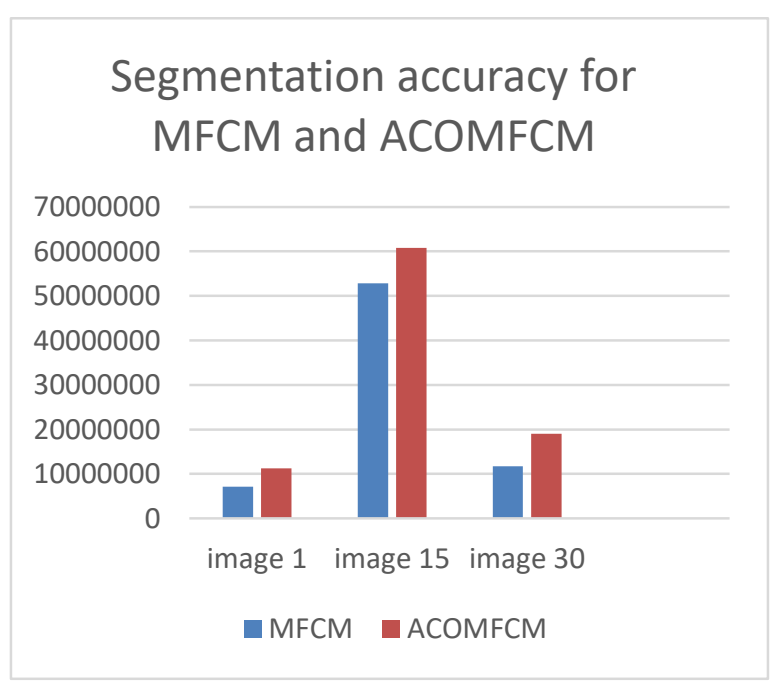

Figure 4.5: Segmentation accuracy for MFCM and ACOMFCM

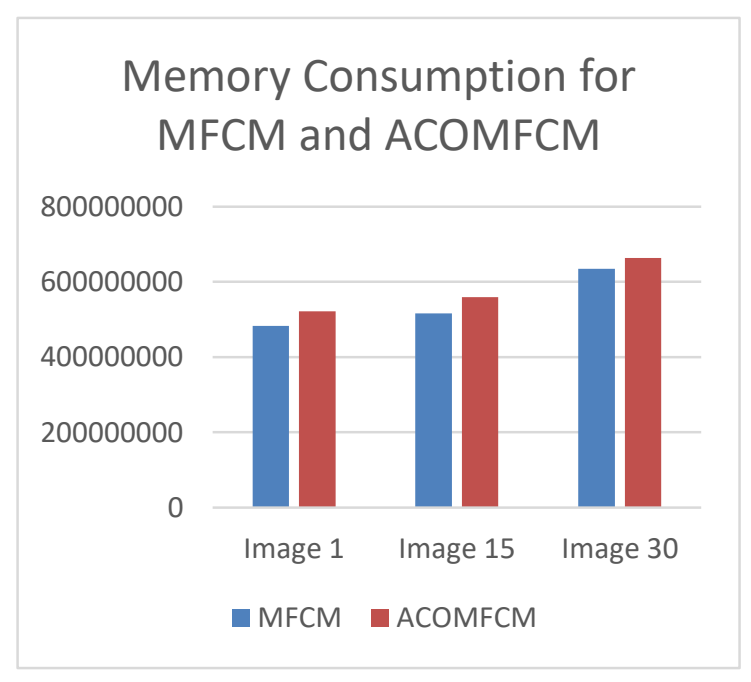

Figure 4.6: Memory consumption for MFCM and ACOMFCM

\section{Conclusion}

This work combined Ant colony optimization (ACO) with existing modified fuzzy c means segmentation algorithm (MFCM) to develop Ant Colony Optimization with Modified Fuzzy CMeans segmentation (ACOMFCM) algorithm. Performance evaluation of the existing MFCM and the developed ACOMFCM was done using segmentation accuracy, segmentation time and memory consumption.

The result shows that ACOMFCM algorithm performed better than the existing MFCM algorithm because it produces higher segmentation accuracy and lower segmentation time.This work will assist the medical personnel in effective medical image diagnosis. 


\section{References.}

Ajala, Oke, Adedeji, Alade, Adewusi (2012). Fuzzy k-c-means Clustering Algorithm for Medical Image Segmentation.Journal of Information Engineering and Applications.Vol 2, No.6, pp 21-32

Cheraghali, Amin, Mohammed, Hossein and Moazameh (2014)."A trading partnership model for estimating discharge permits in river system by Ant Colony Optimization”. Journal of Applied Research in waste water. 2(5):86-92.

Dasgupta, (2012). Demarcation of Brain Tumor Using Modified Fuzzy C-Means. International Journal of Engineering Research and Applications (IJERA) ISSN: 2248-9622 Vol. 2, Issue 4, July-August 2012, pp.529-533

Ghizlane, Amina and Khalid (2013). "Assignment of container trucks of a road transport company with consideration of load balancing pattern". International Journal of Computer Science and applications. 10(2):1-14

Hammed and Hadi (2012). "A Modified Fuzzy C-means clustering with spatial information for image segmentation". International Journal of Computer theory and Engineering. 4(5):762-766.

Joyjit, Arun, Himadri and Ashish (2014). "Segmentation techniques using Image recognition and SAR image processing”. IOSR-Journal of Computer Engineering (IOSR-JCE). $6(3): 20-34$

Krishnan and Ramamoorthy (2014) "Fuzzy clustering based Ant Colony Optimization algorithm for MR Brain Image Segmentation”. Journal of Theoretical and Applied Information Technology”. 65(3):644-647.

Lauren, O.D (2001). "Semi-Automatic Medical Image Segmentation. Unpublished M.Tech Thesis, Massachusetts institute of Technology, Massachusetts".

Lucia Ballerni, Leonardo Bocchi and Carina Johansson (2013).“Clustering algorithm for Color image segmentation”.Journal of Modern Engineering Research, (JMER) 3(5):260-269.

Meenaz, Shraddha and Anuradha (2014). "Image Segmentation techniques and its applications for knee joints: A Survey". Journal of Electronics and Communication Engineering, (IOSR-JECE). 9(5):23-28.

Nitesh Sharma, Sheenam and Amit (2014). "Image Segmentation and Medical Diagnosis: A Review". International Journal of Engineering Trends and Technology, (IJETT). 12(2):94-99 
Rubal Ahuja and Krishan Kumar (2014). “A Survey of Image Enhancement Processing using Kmeans". JCDM, College of Engineering. 4(2):421-427.

Sassibala and Sudhavani (2016). "Image Segmentation Techniques: A Review". International Journal of Advanced Research in Electronics and Communication Engineering, IJARECE. 5(5):1844-1859.

Yu, Lee and Jeon (2014). “An adaptive ACO-based Fuzzy clustering algorithm for noisy image segmentation”. International Journal of Innovative Computing, Information and Control. 8(6):3907-3918. 